\title{
Direct estimation of functionals of density operators by local operations and classical communication
}

\author{
Carolina Moura Alves, ${ }^{1,2, \text { ๑ }}$ Paweł Horodecki, ${ }^{3}$ Daniel K. L. Oi, ${ }^{2}$ L. C. Kwek, ${ }^{4}$ and Artur K. Ekert ${ }^{2,5}$ \\ ${ }^{1}$ Claredon Laboratory, University of Oxford, Parks Road, Oxford OX1 3PU, U.K. \\ ${ }^{2}$ Centre for Quantum Computation, DAMTP, University of Cambridge, Wilberforce Road, Cambridge CB3 0WA, U.K. \\ ${ }^{3}$ Faculty of Applied Physics and Mathematics, Technical University of Gdańsk, 80-952 Gdańsk, Poland. \\ ${ }^{4}$ Department of Natural Sciences, National Institute of Education, \\ Nanyang Technological University, 1 Nanyang Walk, Singapore 637616 \\ ${ }^{5}$ Department of Physics, National University of Singapore, 2 Science Drive 3, Singapore 117542.
}

(Dated: February 1, 2008)

\begin{abstract}
We present a method of determining important properties of a shared bipartite quantum state, within the "distant labs" paradigm, using only local operations and classical communication (LOCC). We apply this procedure to spectrum estimation of shared states, and locally implementable structural physical approximations to incompletely positive maps. This procedure can also be applied to the estimation of channel capacity and measures of entanglement.
\end{abstract}

PACS numbers: 03.67.-a,03.67.Hk,03.67.Mn

There are many scenarios in quantum information science where it is necessary to estimate certain properties of a quantum state $\varrho$, such as its spectrum, purity or degree of entanglement. Moreover, such estimations are often needed when $\varrho$ is a bipartite state $\varrho_{A B}$, shared by two distant parties, Alice and Bob, who can perform only local operations and communicate classically (LOCC). The desired properties can be then estimated either by resorting to quantum state tomography [1] or more directly, e.g. via estimating non-linear functionals of $\varrho_{A B}$. The second method has the natural advantage of being more efficient, since we compute directly the desired properties without estimating any superfluous parameters. In fact the direct estimation has been successfully applied to local spectrum estimation [2], entanglement detection [2, 3] and the evaluation of one-qubit quantum channel capacities 44. However, the LOCC version of these techniques was left as an open problem. In this paper we show that the two basic techniques, namely, the estimation of nonlinear functionals of quantum states and constructions of Structural Physical Approximations 2, 3] admit LOCC implementation. This opens the possibility of the direct estimation of entanglement and some channel capacities using only LOCC.

As a general remark, let us recall that a quantum operation $\Lambda$ can be implemented using LOCC if it can be written as a convex sum

$$
\Lambda=\sum_{k} p_{k} A_{k} \otimes B_{k}
$$

where $A_{k}$ acts on the subsystem at Alice's location and $B_{k}$ on the subsystem at Bob's location, and $p_{k}$ represent the respective probabilities.

Let us start with the estimation of non-linear functionals of $\varrho_{A B}$ using quantum interferometry. Consider a typical set-up for single qubit interferometry, conveniently expressed in terms of quantum gates and net-

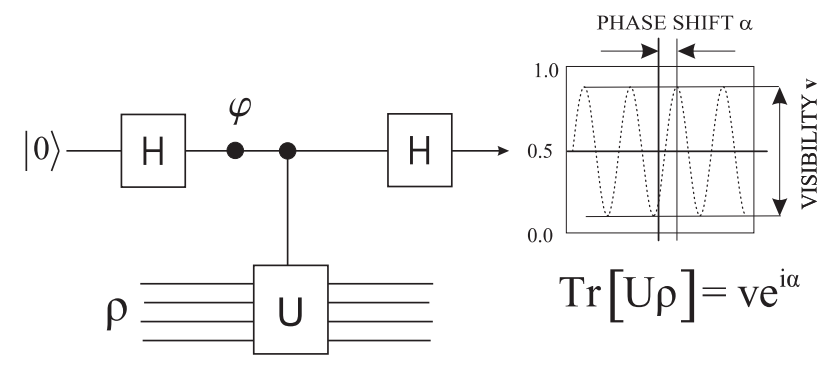

FIG. 1: A modified Mach-Zender interferometer with coupling to an ancilla by a controlled- $U$ gate. The interference pattern is modified by the factor $\mathrm{v} e^{i \alpha}=\operatorname{Tr}[U \rho]$.

works: Hadamard gate, phase-shift gate, Hadamard gate, and measurement in the computational basis $\{|0\rangle,|1\rangle\}$. We modify the interferometer by inserting a controlled$U$ operation between the Hadamard gates, with its control on the qubit and with $U$ acting on a quantum state $\rho$ (Fig. 1). The controlled- $U$ models the interaction between the qubit and an auxiliary system (ancilla), initially in the state $\rho$, and it leads to modification of the observed interference pattern, by the factor $\mathrm{v} e^{i \alpha}=\operatorname{Tr}[U \rho]$. The factor $\mathrm{v}$ is the new visibility and $\alpha$ is the shift of the interference fringes, known as the Pancharatnam phase [6]. The observed modification of the fringes gives us an estimate of the average value of unitary operator $U$ in state $\rho$ [7].

Suppose now that $\rho$ is the quantum state of two separable subsystems, $\rho=\varrho_{A} \otimes \varrho_{B}$ and that we choose $U$ to be the swap operator $V$, defined such that $V|\phi\rangle_{A}|\psi\rangle_{B}=$ $|\psi\rangle_{A}|\phi\rangle_{B}, \forall|\phi\rangle,|\psi\rangle$. In this case, the modification of the interference pattern will be $\mathrm{v}=\operatorname{Tr}\left[V\left(\varrho_{A} \otimes \varrho_{B}\right)\right]=$ $\operatorname{Tr}\left[\varrho_{A} \varrho_{B}\right]$, or the overlap between the input states $\varrho_{A}$ and $\varrho_{B}$. If the two inputs states are equal, $\varrho_{A}=\varrho_{B}=\varrho$, we obtain an estimation of the purity, $\operatorname{Tr}\left[\varrho^{2}\right]$. The gen- 


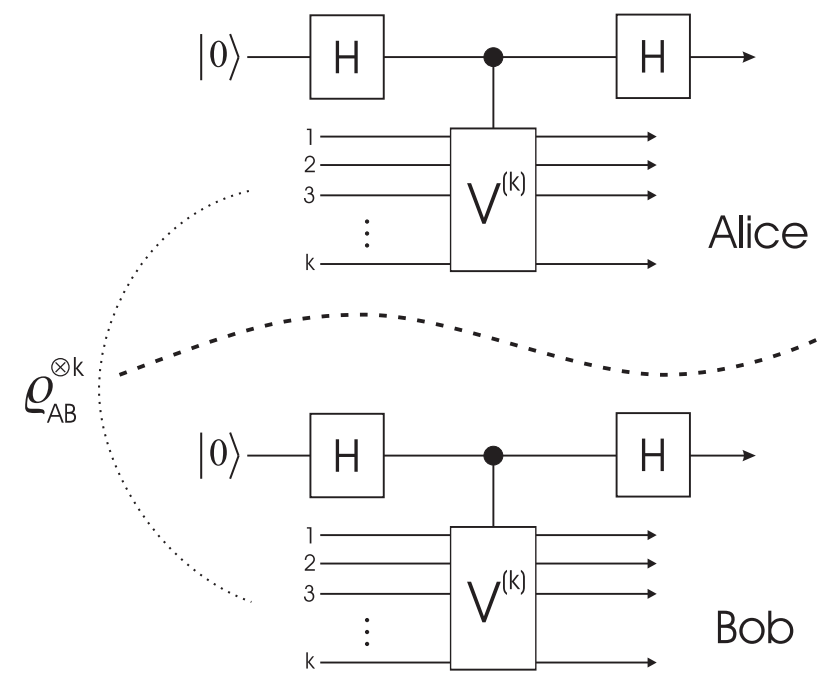

FIG. 2: Network for remote estimation of non-linear functionals of bipartite density operators. Since $\operatorname{Tr}\left[V^{(k)} \varrho^{\otimes k}\right]$ is real, Alice and Bob can omit their respective phase shifters.

eralization of the swap operation $V$ to the shift operation $V^{(k)}\left(V^{(k)}\left|\phi_{1}\right\rangle\left|\phi_{2}\right\rangle \ldots\left|\phi_{k}\right\rangle=\left|\phi_{k}\right\rangle\left|\phi_{1}\right\rangle \ldots\left|\phi_{k-1}\right\rangle, \forall\left|\phi_{i}\right\rangle\right.$, $i=1, \ldots, k)$, and the choice of $\rho=\varrho^{\otimes k}$ as the input state, allows us to estimate multi-copy observables, $\operatorname{Tr}\left[\varrho^{k}\right]$, of an unknown state $\varrho$ [2, 3, 4].

Let us now extend this method to the LOCC scenario by constructing two local networks, one for Alice and one for Bob, in such a way that the global network is similar to the network with the controlled-shift. Unfortunately, the global shift operation $V^{(k)}$ cannot be implemented using only LOCC, since it does not admit decomposition (11). Thus, we will implement it indirectly, using the global network shown in Fig. 2. Alice and Bob share a number of copies of the state $\varrho_{A B} \in \mathcal{B}\left(\mathcal{H}^{d}\right)$. They group them respectively into sets of $k$ elements, and run the local interferometric network on their respective halves of the state $\rho_{A B}=\varrho_{A B}^{\otimes k}$. For each run of the experiment, they record and communicate their result.

The individual interference patterns Alice and Bob record will depend only on their respective reduced density operators. Alice will observe the visibility $\mathrm{v}_{A}=$ $\operatorname{Tr}\left[\varrho_{A}^{k}\right]$ and Bob will observe the visibility $\mathrm{v}_{B}=\operatorname{Tr}\left[\varrho_{B}^{k}\right]$. However, if they compare their individual observations, they will be able to extract information about the global density operator $\varrho_{A B}$, e.g. about

$$
\operatorname{Tr}\left[\varrho_{A B}^{k}\right]=\operatorname{Tr}\left[\varrho_{A B}^{\otimes k}\left(V_{A}^{(k)} \otimes V_{B}^{(k)}\right)\right] .
$$

This is because Alice and Bob can estimate the probabilities $\mathrm{P}_{i j}$ that in the measurement Alice's interfering qubit is found in state $|i\rangle_{A}$ and Bob's in state $|j\rangle_{A}$ for $i, j=0,1$. These probabilities can be conveniently expressed as

$$
\mathrm{P}_{i j}=\frac{1}{4} \operatorname{Tr}\left[\varrho_{A B}^{\otimes k}\left(\mathbb{I}+(-1)^{i} V_{A}^{(k)}\right) \otimes\left(\mathbb{I}+(-1)^{j} V_{B}^{(k)}\right)\right],
$$

hence the formula for the basic non-linear functional of $\varrho_{A B}$ reads

$$
\operatorname{Tr}\left[\varrho_{A B}^{k}\right]=\mathrm{P}_{00}-\mathrm{P}_{01}-\mathrm{P}_{10}+\mathrm{P}_{11} .
$$

In fact, the expression above is the expectation value $\left\langle\sigma_{z} \otimes \sigma_{z}\right\rangle$, measured on Alice's and Bob's qubits (the two qubits that undergo interference). Given that we are able to directly estimate $\operatorname{Tr}\left[\varrho_{A B}^{k}\right]$ for any integer value of $k$, we can estimate the spectrum of $\varrho_{A B}$ without resorting to a full state tomography.

We next show how to implement Structural Physical Approximations within the LOCC constraint. Structural Physical Approximations (SPAs) were introduced recently as tools for determining relevant parameters of density operators (see [2, 3] for more details). Basically the SPA of a mathematical operation $\Lambda$, denoted as $\tilde{\Lambda}$, is a physical operation, a process that can be carried out in a laboratory, that emulates the character of $\Lambda$. More precisely, suppose $\Lambda: \mathcal{B}\left(\mathcal{H}^{d}\right) \mapsto \mathcal{B}\left(\mathcal{H}^{d}\right)$ is a trace preserving map which does not represent any physical process, for example, an anti-unitary operation such as transposition. Then a convex sum

$$
\tilde{\Lambda}=\alpha \mathcal{D}+(1-\alpha) \Lambda,
$$

where $\mathcal{D}$ is the depolarizing map which sends any density operator into the maximally mixed state, represents a physical process, i.e. a completely positive map, as long as $\alpha$ is sufficiently large. On top of this $\mathcal{D}$, with its trivial structure, does not mask the structure of $\Lambda$. The Structural Physical Approximation to $\Lambda$ is obtained by selecting, in the expression above, the threshold value $\alpha=\left(d^{2} \lambda\right) /\left(d^{2} \lambda+1\right)$, where $-\lambda$ is the lowest eigenvalue of $(\mathbb{I} \otimes \Lambda) P_{+}^{(d)}$ and $P_{+}^{(d)}$ is a maximally entangled state of a $d \times d$ system [14].

Please note that the physical implementation of SPAs is not a trivial problem as the formula (5), which explicitly contains the physically impossible map $\Lambda$, is of little guidance here. Let us also mention in passing that if $\Lambda$ is not trace preserving then $\tilde{\Lambda}$ may be implementable but only in a probabilistic sense e.g. via a post-selection.

There are many examples of mathematical operations which although important in the formulation of the physical theory do not represent a physical process. For example, mathematical criteria for entanglement involve positive but not completely positive maps [5] and as such they are not directly implementable in a laboratory they tacitly assume that a precise description of a quantum state of a physical system is given and that such operations are performed on the mathematical description of the state rather than the system itself.

If $\Lambda$ does not represent any physical process then its trivial extension to a bipartite case, $\mathbb{I} \otimes \Lambda$, does not represent a physical process either. Still, its $\mathrm{SPA}, \widetilde{\mathbb{I} \otimes \Lambda}$, does describe a physical operation, but can it be implemented with LOCC? 
The positive answer is obtained by putting $\widetilde{\mathbb{I} \otimes \Lambda}$ into the tensor product form (11). Let us start by writing it as

$$
\begin{aligned}
\widetilde{\mathbb{I} \otimes \Lambda} & =\alpha \mathcal{D} \otimes \mathcal{D}+(1-\alpha) \mathbb{I} \otimes \Lambda \\
& =(1-\alpha+\beta) \mathbb{I} \otimes\left(\frac{1-\alpha}{1-\alpha+\beta} \Lambda+\frac{\beta}{1-\alpha+\beta} \mathcal{D}\right) \\
& +(\alpha-\beta)\left(\frac{\alpha}{\alpha-\beta} \mathcal{D}+\frac{-\beta}{\alpha-\beta} \mathbb{I}\right) \otimes \mathcal{D} \\
& =(1-\alpha+\beta) \mathbb{I} \otimes \tilde{\Lambda}+(\alpha-\beta) \tilde{\Theta} \otimes \mathcal{D},
\end{aligned}
$$

where

$$
\begin{aligned}
\tilde{\Lambda} & =\frac{1-\alpha}{1-\alpha+\beta} \Lambda+\frac{\beta}{1-\alpha+\beta} \mathcal{D}, \\
\tilde{\Theta} & =\frac{\alpha}{\alpha-\beta} \mathcal{D}+\frac{\beta}{\alpha-\beta}(-\mathbb{I}) .
\end{aligned}
$$

Equation (6) does not represent a convex sum of physically implementable maps for any values of $\alpha$ and $\beta$ but if we choose

$$
\begin{array}{r}
\beta \geq(1-\alpha) \lambda d^{2} \\
\alpha \geq \beta d^{2},
\end{array}
$$

where $-\lambda$ is the minimum eigenvalue of $\mathbb{I} \otimes \Lambda\left(P_{+}^{d}\right)$, then indeed $\widetilde{\mathbb{I} \otimes \Lambda}$ is a physical operation in the LOCC form. Note, however, that the map $\tilde{\Theta}$ is not trace preserving and as such it can be implemented only with a certain probability of success. The minimal parameters $\alpha$ and $\beta$ that satisfy inequalities Eqs. (9) and(10) are

$$
\begin{gathered}
\alpha=\frac{\lambda d^{4}}{\lambda d^{4}+1}, \\
\beta=\frac{\lambda d^{2}}{\lambda d^{4}+1} .
\end{gathered}
$$

Hence, the SPA $\widetilde{\mathbb{I} \otimes \Lambda}$ can be implemented, by Alice and Bob, using only only LOCC.

The SPAs have been employed to test for quantum entanglement 22. Recall that a necessary and sufficient condition for a bi-partite state $\varrho_{A B}$ to be separable is $\mathbb{I} \otimes$ $\Lambda\left(\varrho_{A B}\right) \geq 0$, for all positive maps $\Lambda[\underline{5}]$. This condition, when considering the SPA $\widetilde{\mathbb{I} \otimes \Lambda}$ on $\varrho_{A B}$, is equivalent to

$$
[\widetilde{\mathbb{I} \otimes \Lambda}] \varrho_{A B} \geq \frac{d^{2} \lambda}{d^{4} \lambda+1}
$$

where $-\lambda$ is the minimal eigenvalue of the state $[(\mathbb{I} \otimes \mathbb{I}) \otimes(\mathbb{I} \otimes \Lambda)]\left(P_{+}^{d^{2}}\right)[2]$. Thus, by estimating the spectrum (or the lowest eigenvalue) of the state $[\widetilde{\mathbb{I} \otimes \Lambda}] \varrho_{A B}$, we can directly detect quantum entanglement. Moreover, we have already shown that both $\widetilde{\mathbb{I} \otimes \Lambda}$ and the spectrum estimation of $\varrho_{A B}$, via non-linear functionals, can be performed using only LOCC, hence a direct detection of quantum entanglement within the LOCC scenario is also possible.
Let us now comment briefly on other potential applications of the methods presented above. Let a completely positive map $\Lambda: \mathcal{B}\left(\mathcal{H}^{d}\right) \mapsto \mathcal{B}\left(\mathcal{H}^{d}\right)$ represent a quantum channel shared by Alice and Bob. An estimation of the channel capacity may involve either a channel tomography or a direct estimation. In the case of tomography Alice prepares a maximally entangled pair of particles in state $P_{+}^{d}$ and sends one half of the pair to Bob. They now share the state

$$
\varrho_{\Lambda}=[\mathbb{I} \otimes \Lambda] P_{+}^{d} .
$$

From the Jamiołkowski isomorphism [8], this bi-partite state encodes all properties of the channel $\Lambda$, so state tomography on $\varrho_{\Lambda}$ is effectively channel tomography on $\Lambda$. However, given a bi-partite state $\varrho_{\Lambda}$, Alice and Bob can also use the LOCC techniques to directly estimate its desired properties. For example, it has been shown that a single qubit channel $\Lambda$ has non-zero channel capacity if and only if the maximal eigenvalue of $\varrho_{\Lambda}$ is strictly greater than $\frac{1}{2}$ (see [4] for details). This can be estimated directly via the spectrum estimation, which in the case of two qubits requires only $2 \times 4-3=5$ measurements of the type $\sigma_{z} \otimes \sigma_{z}$ as opposed to the 15 parameters required for the state estimation.

For Bell diagonal states (i.e. two-qubit states, whose eigenvectors are all maximally entangled), the entanglement of formation (or negativity, see below) can be inferred from its spectrum [9]. Thus, if Alice and Bob share a Bell entangled state, they can estimate the degree of entanglement of their state through spectrum estimation only. An important subclass of Bell diagonal states are the maximally correlated states, rank two states equivalent (up to $U_{A} \otimes U_{B}$ transformations) to mixtures of two pure states, $\left|\psi_{+}\right\rangle=\frac{1}{\sqrt{2}}(|0\rangle|0\rangle+|1\rangle|1\rangle)$ and $\left|\psi_{-}\right\rangle=\frac{1}{\sqrt{2}}(|0\rangle|0\rangle-|1\rangle|1\rangle)$. The one-way distillable entanglement can be calculated for such states as $D_{\rightarrow}=\log 2-S(\varrho)$, which is a function solely of the spectrum. Thus, instead of estimating the seven parameters required to describe maximally correlated states, we need only estimate five parameters.

The estimation of entanglement measures (see 10] for review) is known only for special cases, such as the computable measure of entanglement 11], $\mathcal{N}\left(\varrho_{A B}\right) \equiv$ $\log \left\|\varrho_{A B}^{T_{B}}\right\|=\log \left(\sum_{i}\left|\lambda_{i}\right|\right)$. This measure is valid for any shared bipartite state, with a maximally mixed reduced density operator of at least one sub-system, and it is a function of the spectrum $\left\{\lambda_{i}\right\}$ of the partially transposed matrix $\varrho_{A B}^{T_{B}} \equiv \mathbb{I} \otimes T\left(\varrho_{A B}\right)$, where $T$ is the (incompletely positive) transposition map. Thus, we can estimate $\mathcal{N}\left(\varrho_{A B}\right)$ using only LOCC, if we choose $\Lambda=T$ and then estimate the spectrum of the resultant state.

Given any quantum channel $\Lambda, \mathcal{N}\left(\varrho_{\Lambda}\right)$ is the upper bound for one-way channel capacity. We obtain, therefore, a necessary condition for non-zero one-way capacity $Q_{\rightarrow}$ : if $\mathcal{N}\left(\varrho_{\Lambda}\right)=0$, the two-way channel capacity must 
vanish [12] (this can be easily seen using distillation and binding entanglement channel [13]). Hence, the positive partial transpose (PPT) test is a strong necessary test of quantum non-zero channel capacity.

To conclude we have demonstrated that both direct spectrum estimations and the structural physical approximations can be implemented in the case of bi-partite states using only local operations and classical communication. This leads to more direct, LOCC type, detections and estimations of quantum entanglement and of some properties of quantum channels. Direct estimations of specific properties have the natural advantage over the state tomography because they avoid estimating superfluous parameters. Still, the exact comparison of the use of physical resources in tomography and direct estimations depends very much on the physical implementations of these techniques. Our objective here is to provide additional tools for quantum information processing rather than comparative studies of these tools.

A.K.E. and L.C.K. acknowledge financial support provided under the A*Star Grant No. 012-104-0040. P.H. acknowledges support from the Polish Committee for Scientific Research and the European Commission. C.M.A. is supported by the Fundação para a Ciência e Tecnologia (Portugal) and D.K.L.O would like to acknowledge the support of the Cambridge-MIT Institute Quantum Information Initiative, and EU projects RESQ (IST-200137559) and TOPQIP (IST-2001-39215).
* Electronic address: carolina.mouraalves@qubit.org

[1] K. Vogel, H. Risken, Phys. Rev. A 40, 2847 (1989)

[2] P. Horodecki, A. Ekert, Phys. Rev. Lett. 89, 127902 (2002).

[3] P. Horodecki, arXiv.org e-print quant-ph/0111036

[4] A. K. Ekert, C. Moura Alves, D. K. L Oi, M. Horodecki, P. Horodecki, L. C. Kwek, Phys. Rev. Lett. 88, 217901 (2002)

[5] M. Horodecki, P. Horodecki, R. Horodecki, Phys. Lett. A 223, 8 (1996)

[6] S. Pancharatnam, Proceedings of the Indian Academy of Science 44, 247 (1956).

[7] E. Sjöqvist, A. K. Pati, A. K. Ekert, J. S. Anandan, M. Ericsson, D. K. L. Oi, V. Vedral, Phys. Rev. Lett. 85, $2845(2000)$

[8] A. Jamiołkowski, Rep. Math. Phys. 3, 275 (1972)

[9] C. H. Bennett, D. DiVincenzo, J. Smolin, W. Wootters, Phys. Rev. A 54, 3824 (1996)

[10] M. Horodecki, Quantum Information and Computation 1, 3 (2001)

[11] R. F. Werner, G. Vidal, Phys. Rev. A 65032314 (2002)

[12] A. S. Holevo, R. F. Werner, Phys. Rev. A 63032312 (2001)

[13] P. Horodecki, M. Horodecki, R. Horodecki, J. Mod. Opt. 47347 (2000)

[14] The threshold value for $\alpha$ is obtained from the requirement of complete positivity of $\tilde{\Lambda}$, which in this case can be reduced to $\tilde{\Lambda} P_{+}^{(d)} \geq 0$ 